\title{
ANALOG COMPUTER FOR STUDYING DIATOMIC MOLECULAR SPECTRA IN TERAHERTZ FREQUENCY
}

\author{
Usman Malik $^{1, a)}$, Muhammad Hamdi 1,b), and Salomo ${ }^{1}$ \\ ${ }^{1}$ Medical Physics and Computation Biophysics, Department of Physics, Universitas Riau, Simpang Baru, \\ Tampan, Pekanbaru City, Riau 28293. \\ Email: ${ }^{a)}$ usman.malik@lecturer.unri.ac.id, ${ }^{b}$ mhdhamdi13@gmail.com
}

\begin{abstract}
This paper introduces a harmonic oscillator model for rovibronic terahertz spectrum of a model of a rigid diatomic rotor with some control parameters. The model shows a study of rotationally-resolved terahertz band spectra of the vibrational transition in diatomic molecules. THz radiation absorption is used as a closed-form system known as the analog computer dynamics mode. The optical terahertz region spectrum of the diatomic molecule consists of a series of lines. Their separations are not exactly constant. A diatomic molecule is not truly a rigid rotator, because it simultaneously vibrates with a small amplitude. Due to quantized vibrational and rotational energy levels and the selection rules, allowed transitions result in a highly ordered spectrum consisting of a $\mathrm{P}$ branch separated by a central gap. Adjacent spectral lines are separated by a spacing of 2B, and since line intensities depend on Boltzmann factor for thermal population and quantum number $J$, each branch monotonically increases and decreases. As temperature increases, more lines are observed, and line intensities decrease due to the population being spread over more rotational levels. Interactivity research also involves on effects of the fundamental vibrational frequency, rotational constant B and temperature included line width on the observed spectrum.
\end{abstract}

Keywords: Diatomic Molecule, Optical THz Region, Rotational-Vibrational Transitions, HarmonicOscillator Model.

\section{INTRODUCTION}

Half of the cosmic background from the Big Bang is in the THz part of the spectrum [1,2,11]. Objects at room temperature emit thermal energy in the optical terahertz range. It is also referred to as submillimeter radiation, terahertz waves, terahertz light, T-lux, and T-rays. The term is generally used for the region of the electromagnetic spectrum between $0.3 \mathrm{THz}$ and $30 \mathrm{THz}$, corresponding to the submillimeter wavelength range of 1 millimeter. The application of T-ray and other forms of radiant energy in life science and medicine can be called the definition of biophotonics. It is also the area of the science that investigates the fundamental interactions of T-ray with biological matter. Tray is used to observe or manipulate biological systems. T-rays have low photon energies $(4 \mathrm{meV}, 1$ $\mathrm{THz}$, one million times weaker than an X-ray photon) and will not cause harmful photoionization in biological tissues. Absorption properties are very different from that of visible light. It can be absorbed by water and many light-transparent organic substances. It is even able to penetrate clothing, paper, and many other opaque materials [1,2,3]. Terahertz radiation is non-ionizing and thus is not expected to damage tissues and DNA. Some frequencies of terahertz radiation can 
penetrate several millimeters of tissue with low water content (e.g., fatty tissue) and is reflected [1,2]. Terahertz radiation can also detect differences in water content and density of tissue [2]. Such methods could allow effective detection of epithelial cancer with a safer and less invasive or painful system using imaging. Some frequencies of terahertz radiation can be used for 3D imaging of teeth and may be more accurate and more reliable than conventional X-ray imaging in dentistry [3,4,5,6]. From $\mathrm{GHz}$ to $\mathrm{THz}$ frequencies, numerous organic molecules exhibit strong absorption and dispersion due to rotational and vibrational transitions $[7,8,11,12,13]$. The interaction of terahertz radiation with biological tissues which is structured from organic atoms and molecules provides an important of probing into their structures. The result of this interaction for a wide range of terahertz spectrum radiation can yield useful bio-molecular information [8,9,10]. For simplicity, this study will be limited to the model of a diatomic molecule in molecular process. The discussion of the quantum mechanical properties in a rigid rotator and a harmonic oscillator is made, which are simple models for molecular rotations and vibrations, respectively. This study is an attempt to discuss rotational and vibrational-rotational spectra in some detail. Frequencies in the range of terahertz will be analyzed and used to study the rotations and vibrations of diatomic molecules. Results of theoretical computation will yield information concerning the mass of the diatomic molecule, stiffness or rigidity of chemical bonds, and vibrational amplitude. Predictions of these models will be compared with experimental studies.

\section{METHODS}

\section{Theoretical Consideration}

The radiation emitted by a black body was due to the oscillations of electrons in the constituent particles of the material body [1] is given by,

$$
\rho(v, T) d v=\frac{8 \pi h v^{3}}{c^{3}} \frac{d v}{e^{h v / k T}-1}
$$

The range of $\mathrm{THz}$ frequencies as a blackbody depicts that the $\mathrm{THz}$ range is room temperature and even it is lower to $10^{\circ} \mathrm{K}$. This temperature region poses the absorption of $\mathrm{THz}$ radiation is due to transitions in an area of rotational energy and also vibrational energy [3]. The energy of rotational and vibrational is possessed by diatomic molecules. In quantum mechanics the Hamiltonian for a harmonic oscillator in spherically symmetric of Laplacian coordinate $\nabla^{2}=\frac{\partial^{2}}{\partial r^{2}}+\frac{2}{r} \frac{\partial}{\partial r}-\frac{\hat{L}}{\hbar^{2} r^{2}}$ is of the form:

$$
\hat{\mathrm{H}}=\frac{\hbar^{2}}{2 \mathrm{~m}} \nabla^{2}+\frac{\mathrm{m} \omega^{2}}{2} \mathrm{r}^{2}
$$

Due to the symmetry of the problem, the solving of the stationary Schrödinger equation is performed by $\hat{\mathrm{H}} \psi_{\mathrm{nlm}}=\mathrm{E}_{\mathrm{n}} \psi_{\mathrm{nlm}}$ in spherical coordinates. $n, l, m$ are quantum numbers characterizing the eigenfunctions. The eigenfunctions of $\hat{L}^{2}$ are the spherical harmonics given by,

$$
\hat{\mathrm{L}}^{2} \mathrm{Y}_{\operatorname{lm}}(\theta, \varphi)=-\hbar^{2} \mathrm{l}(\mathrm{l}+1) \mathrm{Y}_{\mathrm{lm}}(\theta, \varphi)
$$

where the angular-momentum operator $\hat{L}$ contains the derivatives concerning the angles $\theta$ and $\phi$. To separate the angular and radial parts of the wave function $\psi_{n l m}$, it is written in the form 


$$
\psi_{\mathrm{nlm}}(\mathrm{r}, \theta, \varphi)=\frac{\mathrm{R}_{\mathrm{nl}}(\mathrm{r})}{\mathrm{r}} \mathrm{Y}_{\operatorname{lm}}(\theta, \varphi)
$$

Substituting (2) and using (3), (4) yields

$$
\mathrm{R}^{\prime \prime} \mathrm{nl}+\left(\frac{2 \mathrm{mE} \mathrm{nl}}{\hbar^{2}}-\frac{\mathrm{m}^{2} \omega^{2}}{\hbar^{2}} \mathrm{r}^{2}-\frac{\mathrm{l}(\mathrm{l}+1)}{\mathrm{r}^{2}}\right) \mathrm{R} \mathrm{nl}=0
$$

Employing the same arguments as in the case of the linear oscillator, since the solutions are required to be regular at infinity, the hypergeometric function has to break off, leading this time to the condition:

$$
\frac{1}{2}\left(1+\frac{3}{2}-\mathrm{k}\right)=-\mathrm{n}, \quad(n \in N)
$$

i.e., to quantization of energy

$$
\mathrm{E}_{\mathrm{nl}}=\hbar \omega\left(2 \mathrm{n}+\mathrm{l}+\frac{3}{2}\right)
$$

The complete, not yet normalized, eigenfunction belonging to the eigenvalue $E_{n l}$ is then:

$$
\psi_{n l m}(r, \theta, \varphi)=\mathrm{r}^{1} \mathrm{e}^{-(\lambda / 2) \mathrm{r}^{2}} 1 \mathrm{~F}_{1}\left(-\mathrm{n}, 1+3 / 2 ; \lambda \mathrm{r}^{2}\right) \mathrm{Y}_{\mathrm{lm}}(\theta, \varphi)
$$

The $2 l+1$ eigenstates with the same $(n, l)$ but different magnetic quantum numbers $m$ are degenerate. Furthermore, the states with constant $N \equiv 2 n+1$ are degenerate, too. Therefore, $\mathrm{N}$ is sometimes called the principal quantum number. In spectroscopy, it is common to use the notation $s, p, d, f \ldots$ For the angular momenta $l=0,1,2,3, \ldots$.

\section{Analog Computer Circuit Design}

The optical terahertz region is used for investigating the different molecular process. The absorption of terahertz frequency in the range of 0.1 to $30[2,15]$ may be due to the transition between the vibrational or rotational energy level. The absorption of the specific ranges can be due to transitions between rotational or vibrational levels, accompanied by transitions between energy levels. The frequency of the terahertz radiation absorbed is given by $\Delta E=E_{u}-E_{l}$, where $E_{l}$ is the energies of upper and lower states, respectively. Equation (7) gives the allowed energies of a rigid rotator. It shall be proved that the terahertz range can cause a rigid rotator to undergo transitions from one state to another. In particular, based on the selection rule, transitions from only adjacent states are allowed or that $\Delta l= \pm 1$ [3]. In the case of absorption of terahertz spectrum, the molecule goes from a state with a quantum number $l$ to one with $l+1$. The energy difference is:

$$
\Delta \mathrm{E}=\mathrm{E}_{l+1}-\mathrm{E}_{l}=\frac{\hbar^{2}}{2 \mathrm{I}}\left[(\mathrm{l}+1)(\mathrm{l}+2)-(\mathrm{l}(\mathrm{l}+1)]=\frac{\hbar^{2}}{\mathrm{I}}(\mathrm{l}+1)=\frac{\mathrm{h}^{2}}{4 \pi^{2} \mathrm{I}}(\mathrm{l}+1)\right.
$$

The energy levels and the absorption transitions use the Bohr frequency condition $\Delta \mathrm{E}=\mathrm{hv}$, the frequencies at which the absorption transition occurs [1]. A common practice in spectroscopy is to express the transition frequency regarding wavenumbers $\left(\mathrm{cm}^{-1}\right)[5]$ rather than hertz $(\mathrm{Hz})$ 


$$
\overline{\mathrm{v}}=2 \overline{\mathrm{B}}(\mathrm{l}+1) \quad l=0,1,2, \ldots \ldots \ldots
$$

where $\bar{B}$ is the rotational constant expressed in units of wavenumbers. $\bar{B}=\mathrm{h} / 8 \pi^{2} \mathrm{cI}$ is the rotational constant of the molecule. Furthermore, because $I=\mu r^{2}$, where $r$ is the internuclear distance or bond length, one can then determine the bond length. A diatomic molecule makes a transition from one vibration energy state to another by absorbing or emitting terahertz spectrum whose observed frequency satisfies the Bohr frequency condition $\Delta \mathrm{E}=\mathrm{h} v_{\text {obs }}$. The harmonic-oscillator model based on the selection rule allows transitions between adjacent energy states only so that it has the condition $\Delta \mathrm{n}= \pm 1$. For absorption to occur, $\Delta \mathrm{n}= \pm 1$ and so $\Delta \mathrm{E}=\mathrm{E}_{\mathrm{n}+1}-\mathrm{E}_{\mathrm{n}}$. Thus, the observed frequency of terahertz radiation absorbed is:

$$
\overline{\mathrm{V}}_{\text {obs }}=\frac{1}{2 \pi \pi}\left(\frac{\mathrm{k}}{\mu}\right)^{1 / 2}
$$

Where the overbar indicates that the unit is $\mathrm{cm}^{-1}$. Analysis of model which uses the design of analog computer as a simple method for finding energy level of rotational and vibrational molecules, respectively. It can be seen in Figure 1 The main parts of the design are energy levels and wave functions.

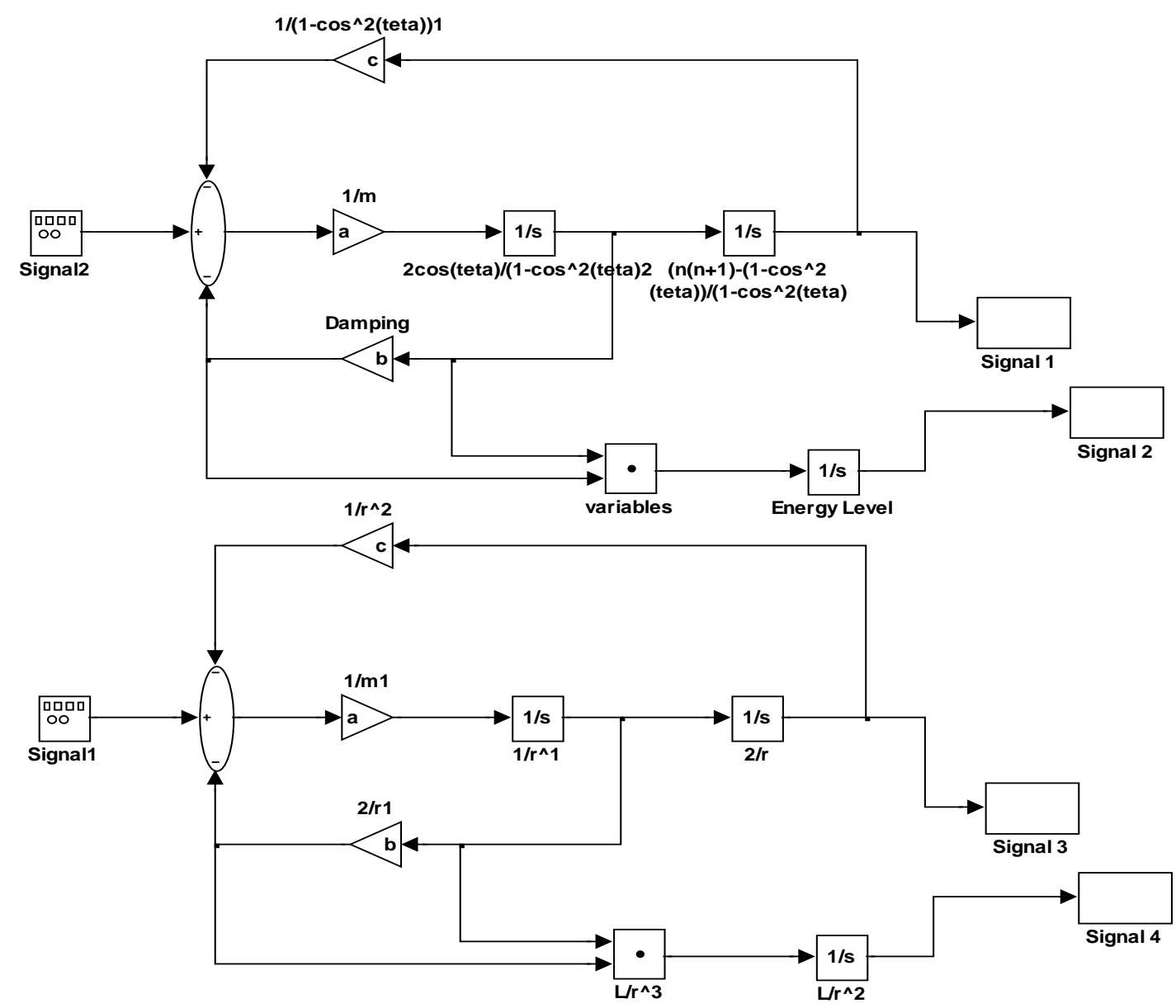

Figure 1. Construction of an analog computer for rotational-vibrational transition in terahertz range circuit. 


\section{RESULTS AND DISCUSSION}

The radiation of the terahertz range can cause diatomic molecules as a rigid rotator to undergo transitions from one state to another. If the atoms can absorb radiation, then the frequencies can identify the diatomic molecules. The transitions occur from only adjacent states are allowed that is, $\Delta l= \pm 1[5,14,16]$. Figure 2 shows the result of the model for the rovibronic terahertz spectrum model of a rigid diatomic rotor with some control parameters. This model shows a result of the study of rotationally-resolved terahertz band spectra of the $\mathrm{v}_{0} \rightarrow \mathrm{v}_{1}$ vibrational transition in diatomic molecules. Due to quantized vibrational and rotational energy levels and the selection rules $\Delta \mathrm{v}= \pm 1$ and $\Delta \mathrm{J}= \pm 1$, allowed transitions result in a highly ordered spectrum consisting of a $\mathrm{P}$ branch $(\Delta \mathrm{J}=-1$, smaller wavenumbers) and an $\mathrm{R}$ branch ( $\Delta \mathrm{J}=+1$, larger wave numbers) separated by a central gap. Adjacent spectral lines are separated by a spacing of 2B. Since line intensities depend on the Boltzmann factor for thermal population and the quantum number $\mathrm{J}$, each branch monotonically increases and/or decreases. As temperature increases, more lines are observed, and the intensities of the lines decrease due to the population being spread over more rotational levels. Interactivity involves the effects of the fundamental vibrational frequency, constant rotational $\mathrm{B}$, temperature, and line width on the observed spectrum.

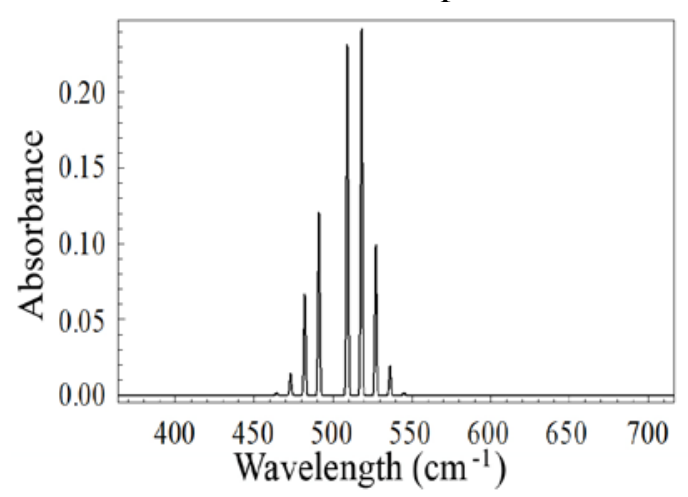

(a)

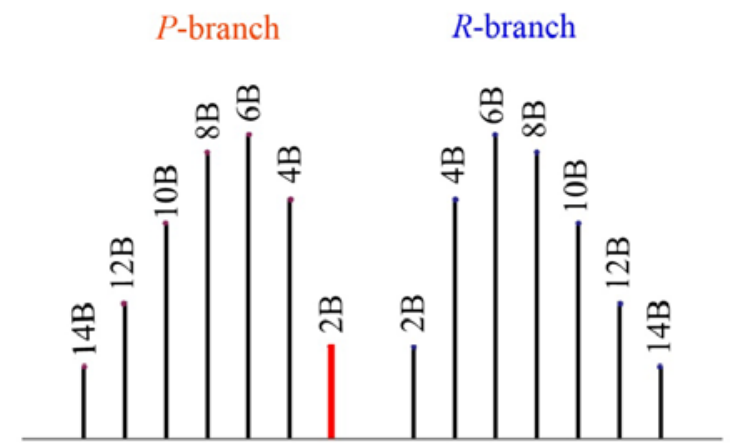

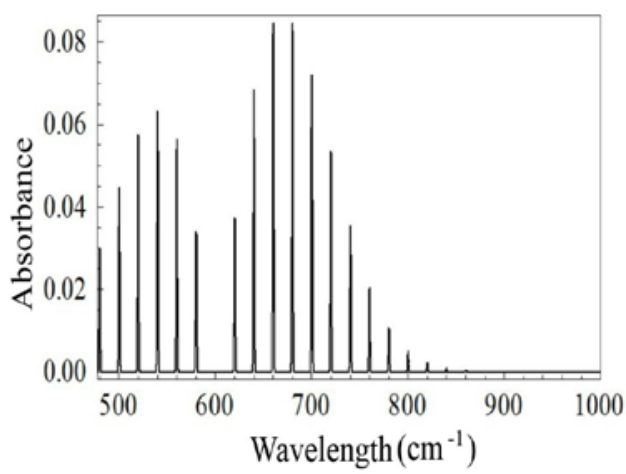

(b)

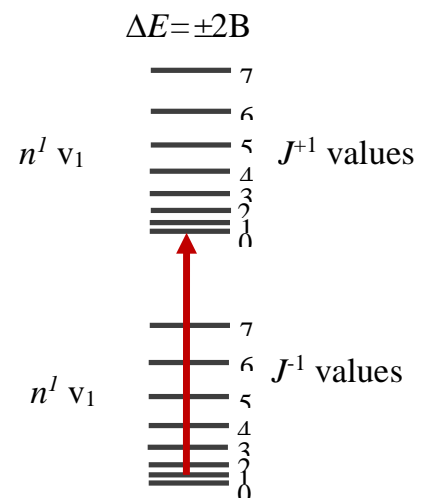

(c)

Figure.2. (a) Rovibronic lowest, (b) highest terahertz spectrum model of a rigid diatomic rotor, and (c) with a transition between $\mathrm{J}^{\prime}=0$ and $\mathrm{J}^{\prime \prime}=1$ (red arrow, $\Delta \mathrm{J}=-1$ ) if $\mathrm{J}^{\prime}=1$ and $\mathrm{J}$ ' $=0$ (blue arrow, $\Delta \mathrm{J}=+1$ )

As can be seen in Figure 2 (a) a result of the model for the lowest fundamental vibration frequency about $500 \mathrm{~cm}^{-1}$, constant rotational $\mathrm{B}, 45 \mathrm{~cm}^{-1}$, Temperature $20 \mathrm{~K}^{\circ}$ and line width $1 \mathrm{~cm}^{-1}$. Whereas Figure 2 (b) with the lowest fundamental vibration frequency is about $900 \mathrm{~cm}^{-1}$, constant rotational $\mathrm{B}, 150 \mathrm{~cm}^{-1}$, temperature $300 \mathrm{~K}^{\circ}$ and line width $1 \mathrm{~cm}^{-1}$. In parameter controls, the model expressions for the simulated spectra assume that the diatomic molecule is a rigid rotator, with a small anharmonicity constant approach zero, zeros electronic angular momentum. The rotational constants of the upper and lower states in any given transition are essentially equal $\left(\mathrm{B}^{\prime \prime} \approx \mathrm{B}^{\prime}\right)$. It is also assumed that only the $\mathrm{v}=0$ vibrational states are thermally populated, to eliminate the presence of "hot bands" and simplify the spectra. In addition to the requirement that $\Delta l= \pm 1$, the molecule must 
also pose a permanent dipole moment. In the case of absorption of radiation, the molecule goes from a state with a quantum number, $l$ to one with $l+1$. The result of the theoretical calculation shows a reduced mass for the diatomic molecule with $\mu$ typically $10^{-25}-10^{-26} \mathrm{~kg}$ and the moment of inertia, $I$ is $10^{-45}-10^{-46} \mathrm{~kg} \cdot \mathrm{m}^{2}$ and the absorbed frequencies are about $2 \times 10^{10}-1 \times 10^{11} \mathrm{~Hz}$. This is still within the range of microwave $[3,12,16]$ having in the rotational transitions of diatomic molecules occurs in the microwave region [5,14]. If this range is compared with the range of $\mathrm{THz}$ light, that is, $0.1-30 \mathrm{THz}$ $[2,14]$, it can be seen that a frequency of $0.1 \mathrm{THz}$ lies in the terahertz region. Consequently, rotational transitions of diatomic molecules can occur in the lowest frequency of the terahertz frequencies range is $0.1 \mathrm{THz}$. This is proved and substantiated by results from experiments, T-ray spectroscopy $[2,16]$.

Figure 3 and 4 show the rigid rotator molecules in all of the numbers model and the experimental data shows good agreement for the moment of inertia and the bond length spectrum. The diatomic molecules consist of a series of equally spaced lines (for the first three rotational quanta). The force constant of diatomic molecules for the terahertz range in all of the numbers infrared regions numbers, $(l=0,1,2)$ with a separation of $2 \mathrm{~B} \mathrm{~Hz}$ or $2 \mathrm{~B} \mathrm{~cm}^{-1}$. From the separation between the absorption frequencies, one can determine the rotational constant and the moment of inertia of the molecule. Furthermore, the bond length of diatomic can be determined from the inertia moment via the internuclear distance. A diatomic molecule is not indeed a rigid rotator, because it vibrates even simultaneously, but small amplitude. Consequently, the result of theoretical computation might be expected that although the terahertz region spectrum of the diatomic molecule consists of a series of lines, their separation is not exactly constant. This model predicts the spectrum associated with a harmonic oscillator consists of just one line.

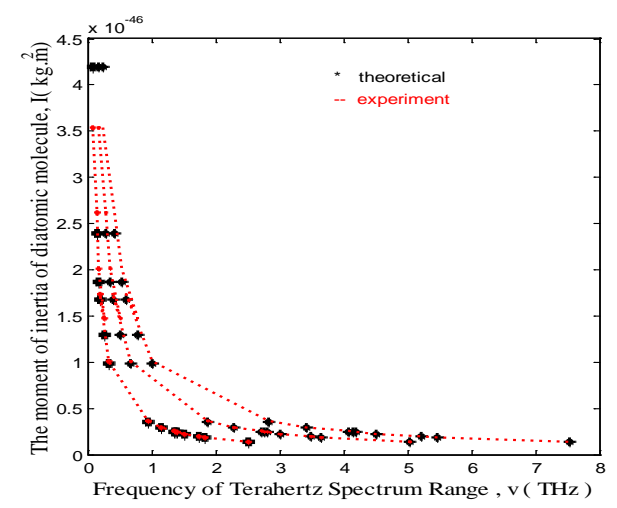

Figure.3. The terahertz spectrum range for a moment of inertia of diatomic molecules in the first three rotational quantum numbers.

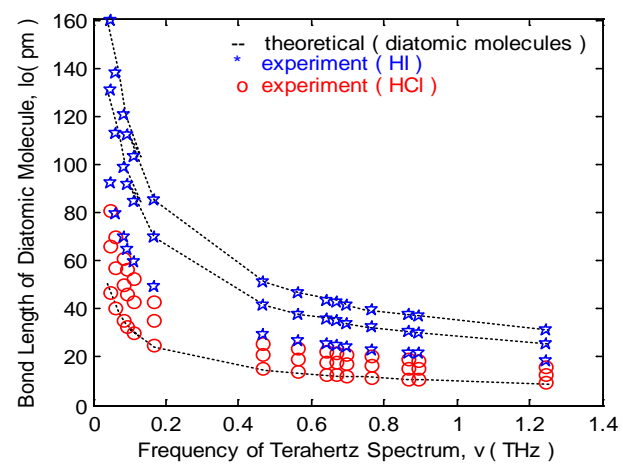

Figure.4. Terahertz spectrum range for the bond length of the diatomic molecule for the first rotational quantum number. 


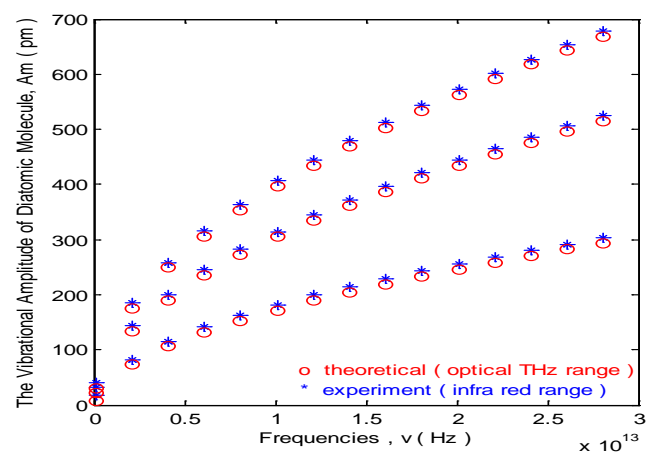

Figure.5. The vibrational amplitude of diatomic molecules in the first three vibrational quanta the numbers.

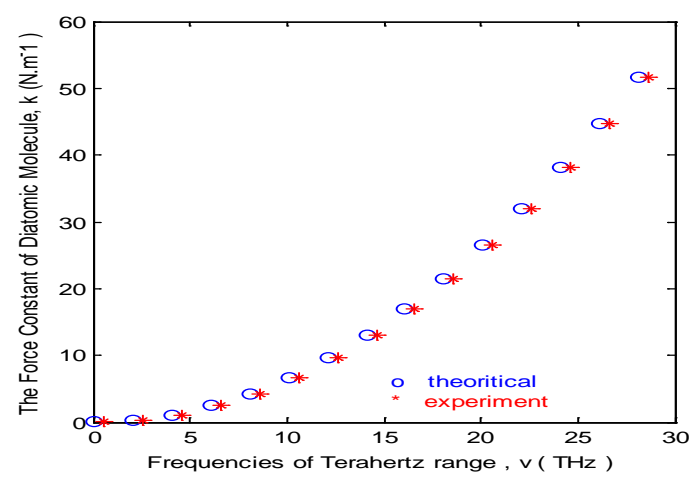

Figure.6. The force constant of diatomic molecules for the terahertz range in all of the number

The vibration of a diatomic molecule indicates a harmonic oscillator. The quantum-mechanical energies are used to describe the terahertz spectrum of a diatomic molecule and to determine the molecular force constants and vibrational amplitudes. Furthermore, because all the energy states are separated by $\hbar \omega, \Delta \mathrm{E}$ is the same for all allowed transitions. The harmonic oscillator model analysis allows one to correct for the fact that the bond is not precisely rigid. We shall also see not only must $\Delta \mathrm{n}= \pm 1$ in the harmonic-oscillator model but also the dipole moment of the molecule must change as the molecule vibrates. Thus, the harmonic-oscillator model predicts that $\mathrm{HCl}$ absorbs in the terahertz spectrum but $\mathrm{N}_{2}$ is not. This result shows in good agreement with experiment. There are, indeed, deviations from the harmonic-oscillator model, not only they are relatively small but also systematically introduce corrections and further modifications.

Figure 5, the first three vibrational quantum numbers show that the vibrational amplitudes $\left(A_{\text {rms }}\right)$ in diatomic molecules. The vibrational spectrum of a diatomic molecule consists of just one line in terahertz range and the frequency of this line is in good accord with experiment. This line is called the fundamental vibrational frequency. These lines occur at around $10^{-3} \mathrm{~cm}^{-1}$ which for diatomic molecules is the terahertz range. For example, $\mathrm{H}^{35} \mathrm{Cl}, v_{\text {obs }}$ is about $2900 \mathrm{~cm}^{-1}$ and $\mathrm{A}_{\mathrm{rms}}=7.67 \times 10^{-12}$ $\mathrm{m}=7.67 \mathrm{pm}$. Figure 6 shows the values of force constants for diatomic molecules in the specific range of the terahertz frequencies for all the infrared regions where for ${ }^{75} \mathrm{Br}^{19} \mathrm{~F}, v_{\text {obs }}=380 \mathrm{~cm}^{-1}$ and $\mathrm{k}$ $=129 \mathrm{Nm}^{-1}$. As can be seen in Figure 6 the force constants for diatomic molecules generally are of the order of $10^{2} \mathrm{~N} \cdot \mathrm{m}^{-1}$.

A small value of $\mathrm{k}$ implies a weak or loose spring, and a large value of $\mathrm{k}$ implies a stiff spring which connects with the chemical bond. The potential is zero at the equilibrium position which indicates that the distance of two molecules equal to the bond length of the molecule.Therefore, this suggests that this is a good approximation for vibration with small amplitudes. 


\section{CONCLUSION}

The interaction of terahertz radiation with molecules in spectroscopy process is a rich source of information concerning the size, shape, and electron distribution within molecules. The simplest model of the rotation and the vibrations of molecules are the rigid-rotator models and the harmonicoscillator model. The rigid-rotator model predicts that the rotation spectrum of a diatomic or linear molecule consists of a series of equally spaced lines in the optical terahertz region. The spacing of these lines gives the bond lengths in the molecule. Although the lines in the terahertz spectrum of a molecule are almost equally spaced, closer examination shows that the lines are not quite equally spaced. The modification of the rigid rotator model by including centrifugal distortion accounts well for the details of the rotational spectra of diatomic molecules. The harmonic-oscillator approximation predicts that the vibrational spectrum of a diatomic molecule consists of just a single line in the terahertz region. The frequency of this line gives the force constant of the molecule. Closer examination of the terahertz spectrum of the diatomic molecule shows that there are weak overtones, whose frequencies are almost, but not quite, integral multiples of the frequency of the fundamental line. The average bond length increases slightly with vibration energy.

\section{ACKNOWLEDGMENT}

The authors thank a research group of the medical physics and computation Biophysics from the Department of Physics, Faculty of Math and Science University of Riau Pekanbaru Indonesia to support the project.

\section{REFERENCES}

[1] J. Dai and John Z. H. Zhanga, "Quantum adsorption dynamics of a diatomic molecule on surface: Four-dimensional fixed-site model for H2 on Cu(111),” J.Phys., vol. 26, pp. 723726, 2006.

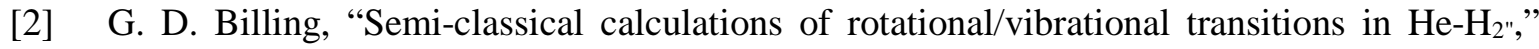
Chemistry Laboratory III, H.C. Ørsted Institute, pp. 534-535, 2010.

[3] U. Thumm et al., "Quantum-beat analysis of the rotational-vibrational dynamics in $\mathrm{D}_{2}{ }^{+}$," American Physical Society, 40th Annual Meeting of the APS Division of Atomic, Molecular and Optical Physics, pp. 123-124, May 2009.

[4] G. Herzberg. "Forbidden Transitions In Diatomic Molecules: V. The Rotation-Vibration Spectrum Of The Hydrogen-Deuteride (Hd) Molecule,” J. Phys., vol. 38, no. 6, pp. 806-818, 2007.

[5] S. Zhang et al., "Experimental demonstration of near-infrared negative-index metamaterials," Phys. Rev. Lett., vol. 95, pp. 137-404, 2005.

[6] S. Yu et al., "Terahertz Spectroscopy and Global Analysis of the Bending Vibrations," Jet Propulsion Laboratory, Pasadena: California Institute of Technology, 2009, pp. 278

[7] X. Zhou et al., "Magic Wavelengths for Terahertz Clock Transitions,“ School of Electronics Engineering \& Computer Science, Beijing: Peking University, 2009, pp. 237

[8] G. Geoffrey, “Quantum Chemestry,” Prentice-Hall of Indian Private Limited New Delhi110001, second Edition, 1992, pp. 134-136, 196-197

[9] R. Alen, "Process of Spectroscopy in Diatomic Molecules," The second edition John Wiley \& Son, 1978, pp. 1256-1257.

[10] B. Norman et al., " Introduction to Infrared and Raman Spectroscopy Third Edition," Academic Press, Inc. Harcourt Brace Jovanovich, 2006. 
[11] Mu-Zi Li and Xue-Bin Bian, "Redshift in High-order harmonic generation from diatomic molecules in on-Born- Oppenheimer approximation”. The 8th International Symposium on Ultrafast Phenomena and Terahertz Waves, OSA, 2016.

[12] G. Z. Kiss et al., "Efficient Numerical Method for Investigating Diatomic Molecules with Single Active Electron Subjected to Intense and Ultrashort Laser Fields,” TIM17 Physics Conference AIP Conf. Proc. vol. 1916, 2017.

[13] Y. Changfeng and W. Zhiwei, "A Universal Analytic Potential Function Applied to Diatomic Molecules," IEEE 3rd International Conference on Communication Software and Networks, pp. 105-110, 2011.

[14] H. A. Abdallah and H. Y. Abdalllah, "Contrastive studies of potential energy functions of some diatomic molecules." The International Conference and Workshop on Basic and Applied Sciences, 2015.

[15] D. V. Chubukov and L. N. Labzowsky, "Corrigendum to " $\Omega$-doubling and a limit for the enhancement of the electron EDM effect in diatomic molecules," Physics Letters A, vol. 379, pp. 803, 2015.

[16] A. Ichihara et al., "Theoretical Study on Isotope-Selective Excitation of Diatomic Molecules by A Terahertz Frequency Comb," Conference on Lasers and Electro-Optics Pacific Rim (CLEO-PR) 2013. 
